\title{
Surface roughness dependence of noncollinear phase matching
}

\author{
O Martinez Matos, G A Torchia, P Vaveliuk, G M Bilmes and \\ J O Tocho
}

Centro de Investigaciones Opticas-CIOp (CONICET-CIC) and Universidad Nacional de La Plata (UNLP), Casilla de Correo 124, 1900, La Plata, Argentina

E-mail: omartin@ciop.unlp.edu.ar

Received 6 June 2001, in final form 28 November 2001

Published 16 January 2002

Online at stacks.iop.org/JOptA/4/130

\begin{abstract}
Spontaneous noncollinear second harmonic generation in potassium dihydrogen phosphate (KDP) crystals was studied theoretically and experimentally as a function of the scattering centres on the surface of the crystal. A model that explains the main features of the second harmonic generated beam was developed in terms of the surface roughness defined by the size of the dispersing grains. It predicts, for the first time, the intensity distribution of the hollow cones obtained in noncollinear phase matching.
\end{abstract}

Keywords: Noncollinear phase matching, second harmonic generation, KDP, Mie scattering

\section{Introduction}

Optical second harmonic generation (SHG), the conversion of radiation $\omega$ into radiation of frequency $2 \omega$, can be produced with high efficiency in certain birefringent crystals achieving phase matching conditions. When the propagation of the generated $2 \omega$ beam is collinear with the propagation of the incident $\omega$ beam, the phenomenon is known as collinear phase matching (CPM). This result is one special case of a most general three-wave interaction in which energy and momentum are conserved. SHG is also possible when two noncollinear beams of frequency $\omega$ are combined to generate a beam of frequency $2 \omega$ in a direction that in general is not collinear with the incident beams. The phenomenon is known as noncollinear phase-matching (NCPM). Normally it is achieved impinging in the crystal with two external light beams inclined on one another to produce a third beam of frequency $2 \omega$. Under particular conditions, if one intense incident beam interacts in the crystal with its own elastic scattered light, that acts as a second wave, the result is a hollow cone of second harmonic light which has been named the spontaneous noncollinear phase matching (SNCPM) effect [1-4].

SNCPM is a phenomenon which has been known for a long time, it was observed for the first time by Giordmaine in 1961 [1] and basically described by Bates [2,3] a few years later. At that time, research was intended to explain the geometrical shape of the second harmonic wave; nearly circular hollow cones were observed and described theoretically. No efforts to obtain the description and distribution of the intensity along the circular cones were made. The influence of the scattering centre was not studied either.

More recently NCPM was proposed to evaluate an optical multiwave-mixing process [4] as well as for the measurement of crystal optical properties. This effect was employed as a three-dimensional nondestructive method to investigate the properties of the illuminated region of the crystal. It was also applied to characterize the optical properties of lithium niobate with and without dopants using the two external beam technique $[5,6]$ and the SNCPM $[7,8]$ method. The SNCPM was also utilized to characterize the changes with temperature in the refractive index of lithium niobate [2]. A geometrical model was developed for the loci of SHG and multiwave-mixing interactions including biaxic crystals; results were compared with experimental data corresponding to NCPM effects in 1-(2thienyl)-3-(4-methyphenyl) propene and (2-furyl) methacrylic anhydride [4]. Other recent work predict the transversal structure of the rings generated when a screen intercepts the hollow cone, showing the typical sinc distribution of the scattering pattern [8], but intensity along the ring was not studied. This paper also shows how the diameter size of the fundamental beam influences the SNCPM ring pattern, reporting that $15 \mu \mathrm{m}$ diameter is a critical value for the ring formation.

Nevertheless, up to now there has been no tri-dimensional model that can explain the intensity distribution in SNCPM as a function of the nonhomogeneity centres that scatter the light. These centres can be in the bulk or in the surface, for instance, 


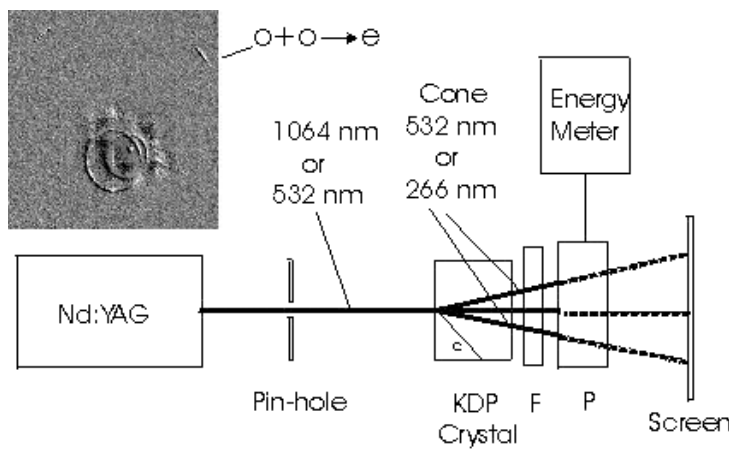

Figure 1. Experimental set-up: $\mathrm{P}$ is a pyroelectric energy meter and $F$ is a filter to remove most of the fundamental beam at 532 or $1064 \mathrm{~nm}$. The photograph corresponds to SNCPM from 1064 to 532 $\mathrm{nm}$; the majority of the internal rings are the product of type-II interactions while the outer rings correspond to type-I interactions ( $\mathrm{o}+\mathrm{o} \rightarrow \mathrm{e}$ means the mixing of two $\omega$ ordinary waves to give an $2 \omega$ extraordinary wave). Results for conversion from 532 to $266 \mathrm{~nm}$ are shown in the following.

in the first work by Giordmaine [1], the cone emission was enhanced by using a lightly ground screen inserted in front of the nonlinear crystal to increase the scattering of light. Natural surface roughness appears as a consequence of the pulling process, due to the attack of water on the surface of hydroscopic materials, or to intrinsic irregularities of the bulk. The granularity of the surface can be controlled, to a relative extent, by polishing, but bulk heterogeneity is much more difficult to avoid. Furthermore, as can be seen later, SNCPM can result in a very simple method to control the quality of the nonlinear crystals.

In this work we measured experimentally SNCPM in potassium dihydrogen phosphate (KDP) crystals with a certain grade of roughness on the surface. Taking into account the size of the dispersing particles of the surface, we develop a model that characterizes and predicts the main properties of the emitted hollow cone; its geometrical shape and size and its two-dimensional distribution intensity are explained in terms of the radiation scattered by the heterogeneity considered.

\section{Experimental details}

A KDP crystal (uniaxial, negative) of cubic shape and $1 \mathrm{~cm}$ side was used for the experiments. The crystallographic axis $(c)$ is at $1.047 \mathrm{rad}$ of one face. Experiments were performed with the second harmonic output of a Q-switched Nd:YAG laser $(532 \mathrm{~nm})$. At this wavelength the phase matching angle $\left(\theta_{P M}\right)$ for type-I SHG is $1.344 \mathrm{rad}$. Due to the fact that the crystal was unpolished for some time, the surface displayed some etching due to water attack. The KDP was mounted in a micrometric system that can rotate and allows one to measure the angle $\theta$ formed between the incident beam and the crystallographic axis with accuracy. The incident beam was limited by a $1 \mathrm{~mm}$ diameter iris. At the output of the KDP, a $532 \mathrm{~nm}$ filter was placed in order to damp the fundamental beam. The energy of the generated double frequency $(\lambda=266 \mathrm{~nm})$ was measured with a pyroelectric detector at the output of the KDP crystal very near to its rear face in order to collect all of the second harmonic generated. The energy measurement was made as a function of the angle $\theta$. At a distance of $2 \mathrm{~m}$ from the crystal a screen of white paper was placed oriented normal to the $532 \mathrm{~nm}$ incident beam; photographs of the screen with the second

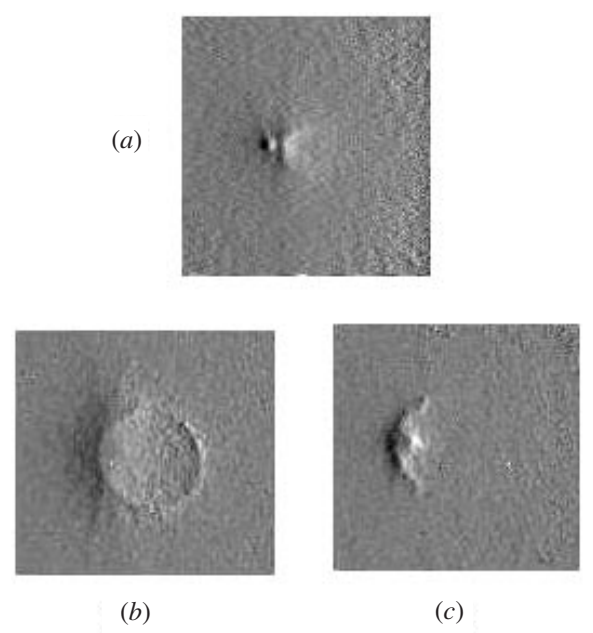

Figure 2. SNCPM from 532 to $266 \mathrm{~nm}$ for different KDP crystal orientation: $(a)$ corresponds to a orientation angle $(\theta=1.338 \mathrm{rad})$ smaller than the CPM angle; $(b)$ is near the CPM $(\theta=1.341)$ and (c) are taken at $\theta=1.346$ rad over the CPM angle.

harmonic pattern were taken for representative angles near the CPM angle. The experimental set-up is shown in figure 1.

Some preliminary studies were made with the $1064 \mathrm{~nm}$ output of the Nd:YAG laser and the KDP crystal oriented to double it. For type-I CPM this corresponds to the crystal oriented at $0.715 \mathrm{rad}$. Type-II phase matching, where the mixing waves have orthogonal polarization, can be obtained at $\theta_{P M}(\mathrm{II})=1.030 \mathrm{rad}$. Under certain conditions SNCPM can be observed simultaneously for both interactions. Furthermore, for type-II NCPM two hollow cones of different size can be produced, resulting in more complex patterns. In a general case, NCPM can be reached for type-I and type-II interactions and three rings are observed on the screen (see the photograph presented in figure 1). This case will be reported in detail in a future work while the remainder of this paper concentrates on the interaction of waves with the same polarization. When $532 \mathrm{~nm}$ is doubled to $266 \mathrm{~nm}$ in KDP, phase matching for type-II interactions is not possible in the collinear case nor in the noncollinear case, either. Only one ring is observed on the screen and results are easy to interpret.

\section{Results}

As the surface of the crystal presents certain roughness, SNCPM are easily observed as a bluish ring of light on the screen when the crystal is oriented near the CPM condition. A brilliant point (BP) of $266 \mathrm{~nm}$ radiation is observed on the screen almost coinciding with the position of the incident $532 \mathrm{~nm}$ beam. Although the shape of the rings are strongly dependent of the orientation of the crystal around the phase matching angle, the position of the BP on the screen is independent of $\theta$.

Changing the angle $\theta$ from a situation far from CPM, to reach first $\theta_{P M}$ and then decreasing this angle, as it is shown in figure 2, the following behaviour is observed. At a certain angle $\theta$ near phase matching, a disc of diffused light appears, while the BP lies outside of it. By increasing $\theta$, the diffused light evolves to form a defined ring (figure $2(a)$ ) with nonuniform intensity along it; the intensity has a minimum at distances far from the BP and a maximum in the side closer to it. These intensity differences are strongly dependent on the size of the 


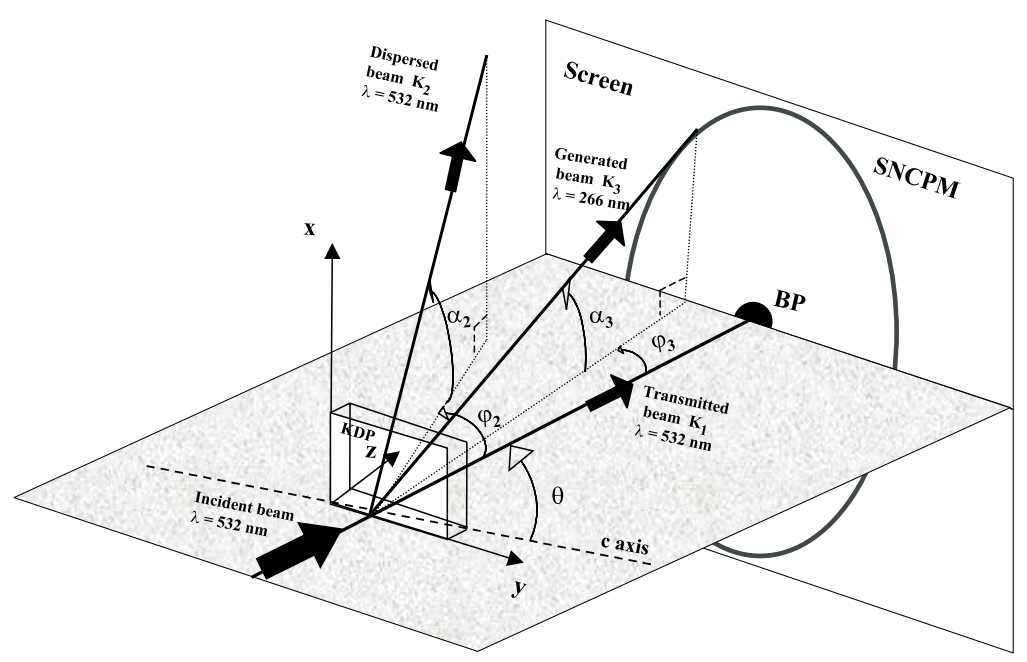

Figure 3. Geometry used to describe SNCPM in the KDP; $\theta$ is the angle between the incidence beam and the crystallographic $c$ axis.

dispersing particles at the surface of the crystal that generated the phenomenon. Increasing $\theta$ again, the diameter of the ring grows and the edge of the maximum intensity becomes closer to the BP (figure $2(b))$. At $\theta_{P M}(1.344 \mathrm{rad})$ this edge overlaps the $\mathrm{BP}$ and the total intensity of the ring reaches a maximum. As $\theta$ goes out from $\theta_{P M}$, the BP falls from this rim, but is now inside the ring, which continues growing in size and decreasing in intensity (figure 2(c)). The thickness of the ring also changes as a function of $\theta$, being wider at the beginning, and thinner at the end of the experiment. The total energy of the hollow cone of the second harmonic wave was measured in terms of the orientation angle $\theta$ using the pyroelectric meter.

\section{The theoretical model for noncollinear phase matching}

To describe the propagation of electromagnetic waves in the KDP crystal, we assume that the three waves can be considered as plane waves. The amplitude of the incident ordinary beam is denoted as $\boldsymbol{E}_{1} ; \boldsymbol{E}_{2}$ is the amplitude of the ordinary part of the radiation dispersed by the scattering centres; $\boldsymbol{E}_{3}$ is the amplitude of the extraordinary generated beam; $\omega_{1}=\omega_{2}=\omega$ the fundamental frequency; $\omega_{3}=2 \omega$ the generated frequency and $\boldsymbol{k}_{i}$ the wavevector associated with each amplitude $\boldsymbol{E}_{i}$ respectively.

Assuming for all the waves that the variation of the amplitudes are very small over distances of the order the wavelength (slowly varying limit approximation $\left.\nabla^{2} \boldsymbol{E} \ll(\boldsymbol{k} \cdot \boldsymbol{r}) \boldsymbol{E}\right)$, the evolution of the waves inside the crystal is similar to the standard ones used for collinear three-wave mixing [9]. It can be obtained in a general vectorial form as

$$
\begin{aligned}
& \left(u_{1} \cdot \nabla\right) \boldsymbol{E}_{1}=\frac{-\kappa_{1}}{2} \boldsymbol{E}_{1}+\frac{\mathrm{i} \omega}{2 c n_{o}^{\omega}} \tilde{\boldsymbol{d}}: \boldsymbol{E}_{3}: \boldsymbol{E}_{2}^{*} \mathrm{e}^{\mathrm{i}(\Delta \boldsymbol{k} \cdot \boldsymbol{r})}, \\
& \left(u_{2} \cdot \nabla\right) \boldsymbol{E}_{2}=\frac{-\kappa_{2}}{2} \boldsymbol{E}_{2}+\frac{\mathrm{i} \omega}{2 c n_{o}^{\omega}} \tilde{\boldsymbol{d}}: \boldsymbol{E}_{3}: \boldsymbol{E}_{1}^{*} \mathrm{e}^{\mathrm{i}(\Delta \boldsymbol{k} \cdot \boldsymbol{r})}, \\
& \left(u_{3} \cdot \nabla\right) \boldsymbol{E}_{3}=\frac{-\kappa_{3}}{2} \boldsymbol{E}_{3}+\frac{\mathrm{i} \omega}{c n_{e}^{2 \omega}} \tilde{\boldsymbol{d}}: \boldsymbol{E}_{1}: \boldsymbol{E}_{2} \mathrm{e}^{-\mathrm{i}(\Delta \boldsymbol{k} \cdot \boldsymbol{r})},
\end{aligned}
$$

where $\boldsymbol{u}_{i}$ are the unitary vectors in the $\boldsymbol{k}_{i}$ direction, $\boldsymbol{\kappa}_{i}$ the linear absorption coefficients, $c$ the speed of light in a vacuum, $n_{o}^{\omega}$ the ordinary refractive index at $\omega$ frequency, $n_{e}^{2 \omega}$ the extraordinary refraction index at $2 \omega$ frequency, $\tilde{\boldsymbol{d}}$ is the nonlinear tensor that allows the combination of frequencies and $\boldsymbol{\Delta} \boldsymbol{k}=\boldsymbol{k}_{3}-\boldsymbol{k}_{2}-\boldsymbol{k}_{1}$ is the momentum mismatch.

For the KDP, the linear absorption in equation (1) can be neglected for both 532 and $266 \mathrm{~nm}$. Assuming small efficiency on frequency conversion, amplitudes $\boldsymbol{E}_{1}$ and $\boldsymbol{E}_{2}$ can be considered constant over the crystal and the system can be solved analytically to obtain the intensity of the second harmonic wave. This expression can be written as

$$
\begin{aligned}
I_{3}= & 2 \frac{\omega^{2}}{c^{2}\left(n_{e}^{2 \omega}\right)^{2}} \frac{\left(\tilde{\boldsymbol{d}}: \boldsymbol{E}_{1}: \boldsymbol{E}_{2}\right) \times\left(\tilde{\boldsymbol{d}}: \boldsymbol{E}_{1}: \boldsymbol{E}_{2}\right)^{*}}{\left(u_{3}\right)^{2}} \\
& \times\left(\frac{\sin (\Delta \boldsymbol{k} \cdot \boldsymbol{r} / 2)}{\Delta \boldsymbol{k} \cdot \boldsymbol{r} / 2}\right)^{2} .
\end{aligned}
$$

If the sizes of the dispersing 'particles' of radius $a$ are large enough compared with the wavelength $(2 \pi a>\lambda)$ we can use the expression given by the scalar theory of the diffraction for the amplitude of the scattered wave in the roughness of the front crystal surface. This result is a good approximation to the more precise but mathematically cumbersome Mie theory [10]:

$$
\boldsymbol{E}_{2}\left(\Gamma_{2}\right)=\frac{\left(\frac{2 \pi a}{\lambda}\right)^{2}}{\boldsymbol{k} \cdot \boldsymbol{r}} \frac{\left(1+\cos \Gamma_{2}\right)}{2} \frac{J_{1}\left(\frac{2 \pi a}{\lambda} \cdot \sin \Gamma_{2}\right)}{\left(\frac{2 \pi a}{\lambda} \cdot \sin \Gamma_{2}\right)},
$$

where $\Gamma_{2}$ is the angle between $\boldsymbol{k}_{1}$ and $\boldsymbol{k}_{2}$ that can be calculated in terms of $\alpha_{2}$ and $\varphi_{2}$ (defined in figure 3) by $\cos \Gamma_{2}=$ $\cos \varphi_{2} \cos \alpha_{2}$.

Now the tensorial products of equation (2) can be solved and the complete expression corresponding to the second harmonic wave intensity in terms of its position on the screen, following the relation of the angles given in figure 3 , can be written as

$$
\begin{aligned}
I_{3}= & 2 \frac{\omega^{2}}{c^{2}\left(n_{e}^{2 \omega}\left(\Gamma_{3}\right)\right)^{2}} \frac{\left(d_{36} f\left(\alpha_{2}, \varphi_{2}, \theta\right)\right)^{2}}{\left(u_{3 z}\right)^{2}} \boldsymbol{E}_{1} \boldsymbol{E}_{1}^{*} \\
& \times\left(\boldsymbol{E}_{2}\left(\Gamma_{2}\right)\right)^{2}\left(\frac{\sin (\Delta \boldsymbol{k} \cdot \boldsymbol{r} / 2)}{\Delta \boldsymbol{k} \cdot \boldsymbol{r} / 2}\right)^{2},
\end{aligned}
$$

where $\Gamma_{3}$ is the angle between the crystallographic axis and the $\boldsymbol{k}_{3}$ vector, and the geometrical relationships with the position $\alpha_{3}, \varphi_{3}$ on the screen and the momentum mismatch, are: 

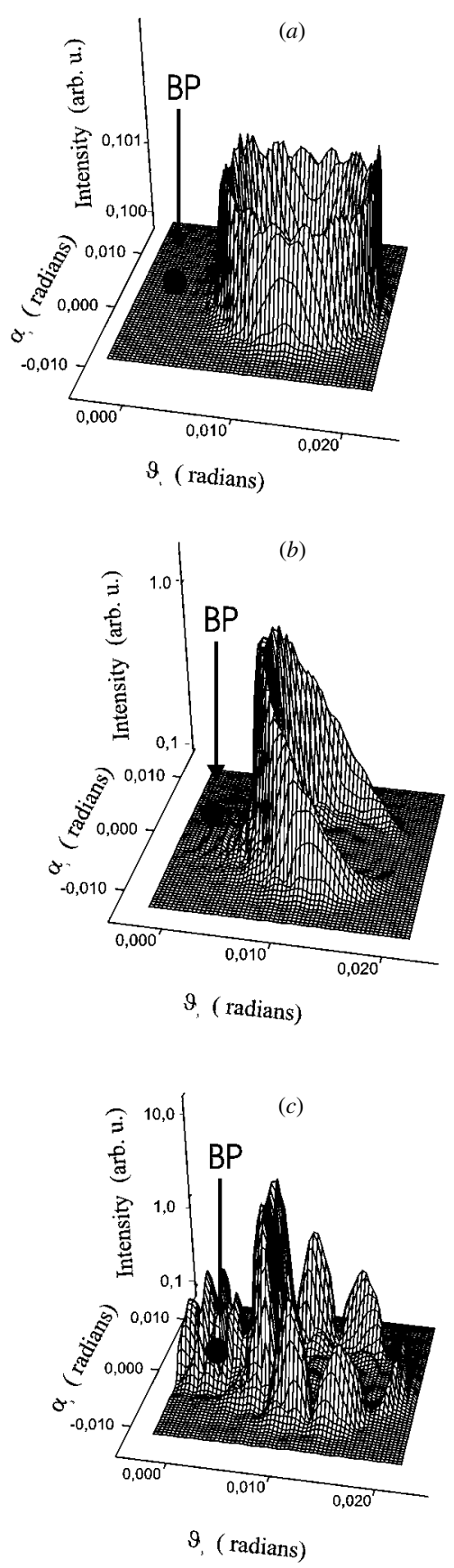

Figure 4. Two-dimensional intensity distribution for different radii of the dispersing particles $1 \mu \mathrm{m}(a), 8 \mu \mathrm{m}(b)$ and $30 \mu \mathrm{m}(c)$.

$f\left(\alpha_{2}, \varphi_{2}, \theta\right)=\frac{\cos \alpha_{2} \sin \left(\theta+\varphi_{2}\right) \sin \theta}{\sqrt{1-\cos ^{2} \alpha_{2} \cos ^{2}\left(\theta+\varphi_{2}\right)}}$,

$\sin \alpha_{2}=\sin \left(2 \alpha_{3}\right) \cos \varphi_{3}$,

$\sin \varphi_{2}=\frac{\cos ^{2} \alpha_{3} \sin \left(2 \varphi_{3}\right)}{\sqrt{1-\sin ^{2}\left(2 \alpha_{3}\right) \cos ^{2} \varphi_{3}}}$

$u_{3 z}=\cos \left(\alpha_{3}\right) \cos \left(\varphi_{3}\right)$,

$\cos \Gamma_{3}=\cos \left(\varphi_{3} \pm \theta\right) \cos \alpha_{3}$,

$\Delta \boldsymbol{k} \cdot \boldsymbol{r}=\frac{\omega}{c}\left[2 n_{e}^{2 \omega}\left(\Gamma_{3}\right) \cos \alpha_{3} \cos \varphi_{3}\right.$

$\left.-n_{o}^{\omega}\left(2 \cos ^{2} \alpha_{3} \cos ^{2} \varphi_{3}-1\right)-n_{o}^{\omega}\right] z$.

The intensity of the NCPM wave given by equation (4) can be compared with the most-used expression for the
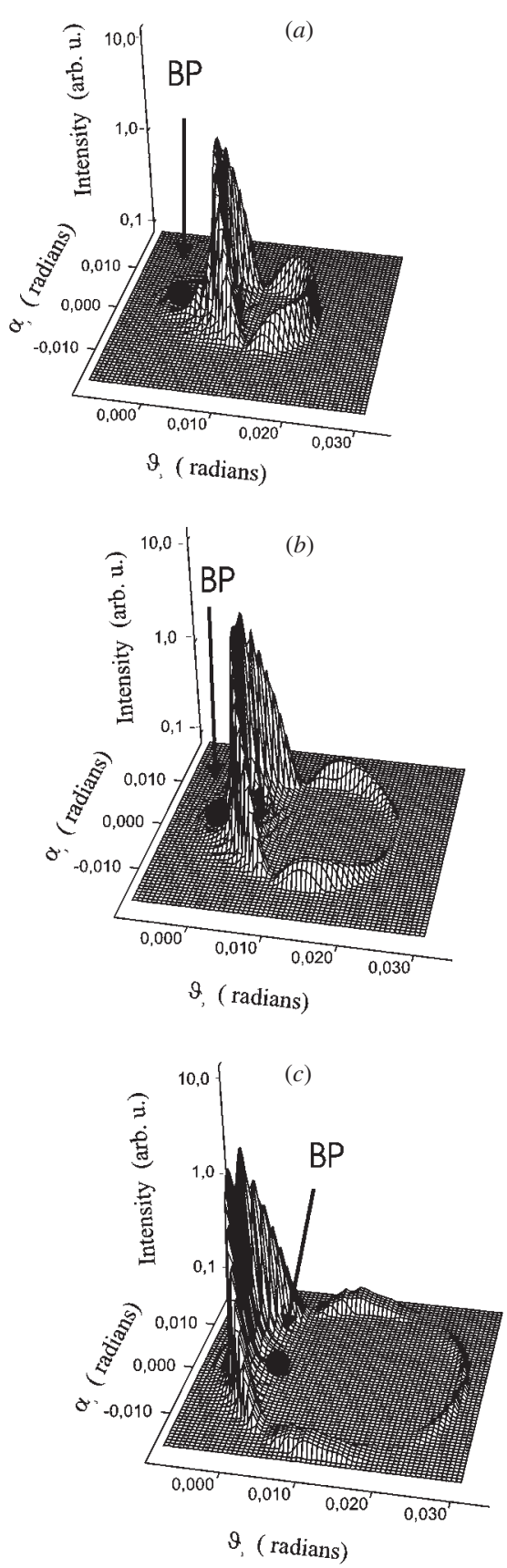

Figure 5. Two-dimensional intensity distribution for $12 \mu \mathrm{m}$ radius scattering particles in terms of the orientation angle $\theta$.

(a) $\theta=1.338 \mathrm{rad} ;(b) \theta=1.341 \mathrm{rad}$ and $(c) \theta=1.346 \mathrm{rad}$.

collinear case,

$$
I_{3}=2 \frac{\omega^{2}}{c^{2}\left(n_{e}^{2 \omega}(\theta)\right)^{2}} \frac{\left(d_{36} \sin \theta\right)^{2} \boldsymbol{E}_{1} \boldsymbol{E}_{1}^{*}}{\left(u_{3}\right)^{2}}\left(\frac{\sin (\Delta \boldsymbol{k} \cdot \boldsymbol{r} / 2)}{\Delta \boldsymbol{k} \cdot \boldsymbol{r} / 2}\right)^{2},
$$

where

$$
\Delta \boldsymbol{k} \cdot \boldsymbol{r}=\frac{2 \omega}{c}\left(n_{e}^{2 \omega}(\theta)-n_{o}^{\omega}\right) z
$$

The position corresponding to the maximum of the intensity distribution corresponds in both cases to $\Delta \boldsymbol{k} \cdot \boldsymbol{r}=0$, and for the noncollinear case it can be written as,

$$
\begin{aligned}
\alpha_{3}= & \pm \arccos \left[\left(\left(-A \cos \varphi_{3}\right.\right.\right. \\
& \left.+\sqrt{A^{2} \cos ^{2} \varphi_{3}+4 B \cos ^{2}\left(\theta+\varphi_{3}\right)}\right)
\end{aligned}
$$




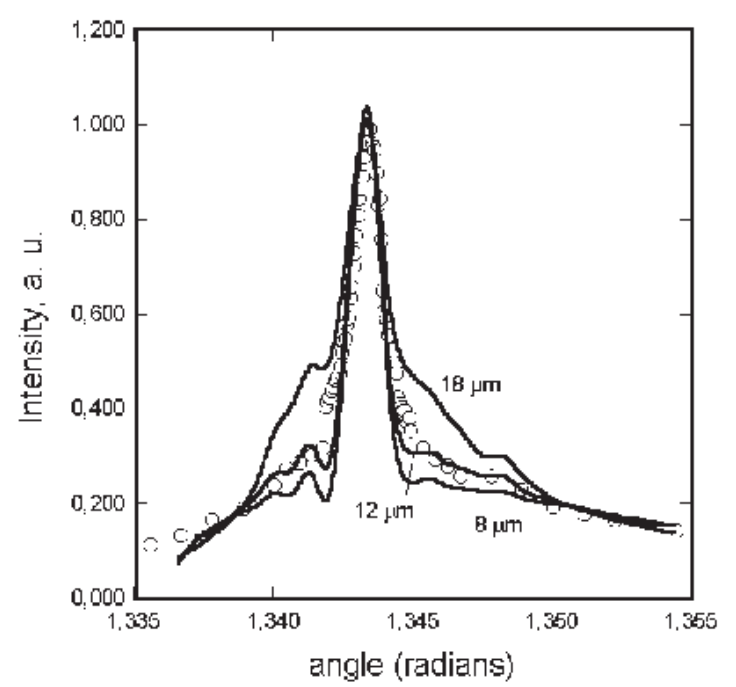

Figure 6. Total intensity of the second harmonic wave in terms the orientation angle. Hollow circles are experimental points measured as explained in the text with the pyroelectric meter. Fittings (solid curves) were calculated for the contributions of the collinear (equation (5)) and noncollinear (equation (4)) phase matching for 8 , 12 and $18 \mu \mathrm{m}$ radius particles; good fitting for both the central part and for the wings is obtained for the $12 \mu \mathrm{m}$ particles.

$$
\begin{aligned}
& \left.\left.\left\{2 B \cos \varphi_{3} \cos ^{2}\left(\theta+\varphi_{3}\right)\right\}^{-1}\right)^{\frac{1}{2}}\right], \\
& \text { with } A=\left(\frac{n_{o}^{(\omega)}}{n_{e}^{(2 \omega)}}\right)^{2} \text { and } \\
& B=\left(\left(\frac{1}{n_{o}^{(2 \omega)}}\right)^{2}-\left(\frac{1}{n_{e}^{(2 \omega)}}\right)^{2}\right)\left(n_{o}^{(\omega)}\right)^{2} .
\end{aligned}
$$

The position of the BP on the screen is given by $\varphi_{3}=0$, $\alpha_{3}=0$. The total second harmonic intensity can be evaluated using equation (4) integrated over the ring.

\section{Ring intensity distribution}

From equation (4) we obtain the two-dimensional ring intensity distribution on the screen, represented in figures 4 and 5. In all cases we used $d_{36}=d_{14}=0.39 \times 10^{-12} \mathrm{~m} \mathrm{~V}^{-1}$ [11] for the KDP. Figure 4 shows the rings intensity distribution for different values of the dispersing particles size, achieved for the same angular position of figure 2(a). The structure depends strongly on the dispersing particle size, showing that the ring intensity is more uniform for small values of $a$. As $a$ increases the ring intensity structure becomes more complex, and new contributions outside the ring appears. This fact can be seen in the early results obtained by Giordmaine [1].

The results given in figure 5 correspond to dispersing particles of $12 \mu \mathrm{m}$ radius with different orientation angles; results indicated as $(a),(b)$ and $(c)$ were obtained for the same $\theta$ angles as the photographs $(a),(b)$ and $(c)$ in figure 2 . We can observe that the BP is outside of the ring in figures $4(a)$ and $(b)$, while in (c) the BP is inside the ring. This fact means that we reach and overcome the PM angles. The ring maximum intensity is always over the side of the BP as can be seen in the photographs. As the $\theta$ value increases, the ring thickness decreases, showing well-defined rings for high $\theta$ values. The model reproduces qualitatively the observed ring intensity behaviour.

\section{The size of the scattering grains}

To further characterize our crystal, the size of the scattering grain obtained by SNCPM, we fitted the total intensity of the ring measured in terms of the orientation angle $\theta$. The total intensity was calculated as the sum of the independent contributions of the NCPM and CPM light intensity. Fittings are shown in figure 6; total intensities (solid curves) were calculated by adding the SNCPM for different particle sizes and the CPM classical distribution of intensities for our conditions. As the last contribution is only important near $\theta_{P M}$ (1.344 rad), the SNCPM intensity was normalized to adjust the experimental results at $1.350 \mathrm{rad}$, in one of the wings; the CPM contribution was added to adjust the maximum at the centre of the distribution. Good fitting for both the wings and the centre of the experimental results was found for particles of $12 \mu \mathrm{m}$ radius. Larger radius values $(18 \mu \mathrm{m})$ give contributions too big for the wings, and particles with smaller size $(8 \mu \mathrm{m})$ produce a thinner distribution.

\section{Conclusions}

The NCPM phenomenon in SHG was characterized as a function of the grain size of the scattering heterogeneities at the surface or in the bulk of noncentrosymmetric crystals. Our theoretical analysis developed here describes the main features of the hollow cones of second harmonic frequency generated. The model explains, for the first time, the intensity distribution along the rings observed in a plane perpendicular to the incident beam.

NCPM was found to be strongly dependent on the size of the heterogeneites. The study of the intensity distribution along the rings and its total intensity relative to the CPM intensity near the phase matching angle, can be used as a simple method to determine the size of the scattering elements, inside or dispersed at the surface, in nonlinear optics crystals. In this way we propose a new real-time method for quality control of the surface of birefringent crystals and we bring a complete model to be used when NCPM is intended as a nondestructive method to characterize the optical properties of the pure crystals and also, to optimize some doping methods $[2,7,8]$.

The presence of NCPM rings can be used also as a simple visual way to achieve the CPM condition. The method to achieve second harmonic maximum efficiencies is to change the orientation angle in order to overlap the rim of maximum intensity of the ring with the brilliant fixed point.

\section{References}

[1] Giordmaine J A 1962 Phys. Rev. Lett. 819

[2] Bates H E 1971 J. Opt. Soc. Am. 617904

[3] Bates H E 1973 J. Opt. Soc. Am. 632146

[4] Zhang G J, Horinouchi S, Kinoshita T and Sasaki K 1995 Appl. Opt. 345301

[5] Reitchert A and Betzler K 1994 Ferroelectrics 15693

[6] Reichert A and Betzler K 1996 J. Appl. Phys. 792209

[7] Schlarb U and Betzler K 1994 Phys. Rev. B 50751

[8] Kasemir K U and Betzler K 1999 Appl. Phys. B 68763

[9] Yariv A 1975 Quantum Electronics 2nd edn (New York: Wiley)

[10] Bohren C F and Huffman D R 1983 Absorption and Scattering of the Light by Small Particles (New York: Wiley)

[11] Dmitriev V G, Gurzadyan G G and Nikogosyan D N 1999 Handbook of Nonlinear Optical Crystals 3rd edn (Berlin: Springer) 